\title{
Swarm Intelligence Approach to QRS Detection
}

\author{
Mohamed Belkadi and Abdelhamid Daamouche \\ Signals and Systems Laboratory, Institute of Electrical and Electronics Engineering, Universite M'Hamed \\ Bougara de Boumerdes, Algeria
}

\begin{abstract}
The QRS detection is a crucial step in ECG signal analysis; it has a great impact on the beats segmentation and in the final classification of the ECG signal. The Pan-Tompkins is one of the first and best-performing algorithms for QRS detection. It performs filtering for noise suppression, differentiation for slope dominance, and thresholding for decision making. All of the parameters of the Pan-Tompkins algorithm are selected empirically. However, we think that the PanTompkins method can achieve better performance if the parameters were optimized. Therefore, we propose an adaptive algorithm that looks for the best set of parameters that improves the Pan-Tompkins algorithm performance. For this purpose, we formulate the parameter design as an optimization problem within a particle swarm optimization framework. Experiments conducted on the 24 hours recording of the MIT/BIH arrhythmia benchmark dataset achieved an overall accuracy of $99.83 \%$ which outperforms the state-of-the-art time-domain algorithms.
\end{abstract}

Keywords: ECG, QRS detection, Pan-Tompkins algorithm, Particle Swarm Optimization.

Received October 9, 2018; accepted November 5, 2019

https://doi.org/10.34028/iajit/17/4/6

\section{Introduction}

The Electrocardiogram (ECG) is widely used by health practitioners for the diagnosis. Recently, it has been used for human authentication [8]. It is a graphical representation of the electro activity of the heart. It comprises three waves, $\mathrm{P}, \mathrm{QRS}$, and $\mathrm{T}$. A great importance is given to the detection of the QRS wave, comprising $\mathrm{Q}, \mathrm{R}$, and $\mathrm{S}$ waves, as it is essential for heart rate determination and beat type recognition and segmentation. The detection of the $\mathrm{R}$ wave, which is the peak of the QRS, is a very difficult task since it has a time-varying morphology and is subject to physiological variations due to the patient state and noise [10]. The RR interval is the interval between two successive $\mathrm{R}$ peaks; identifying $\mathrm{RR}$ intervals permits to separate beats.

Usually, the ECG signal is corrupted by many types of noise that occupy different frequency bands. For instance, $50 / 60 \mathrm{~Hz}$ noise results from power line interference. Patient stress and hyperthyroidism introduce undesirable harmonics in a range of 5-2000 Hz. Other sources of disturbance generate components between 0.02 and $2 \mathrm{~Hz}$. Therefore, any ECG analysis process is preceded by a denoising step.

We find a huge number of methods that deal with the QRS complex detection in the literature. These methods may be divided into three categories, timedomain methods, frequency-domain methods, and timefrequency methods. In this work, we will restrict our focus to the time-domain algorithms. Several timedomain algorithms have been developed in the last three decades. For example, authors in [7] proposed an optimized decision rule process which resulted in improved sensitivity and positive predictivity. In [6], the preprocessing step is different from that one of Pan-Tompkins to cope with the requirements of accuracy and low resource consumption.

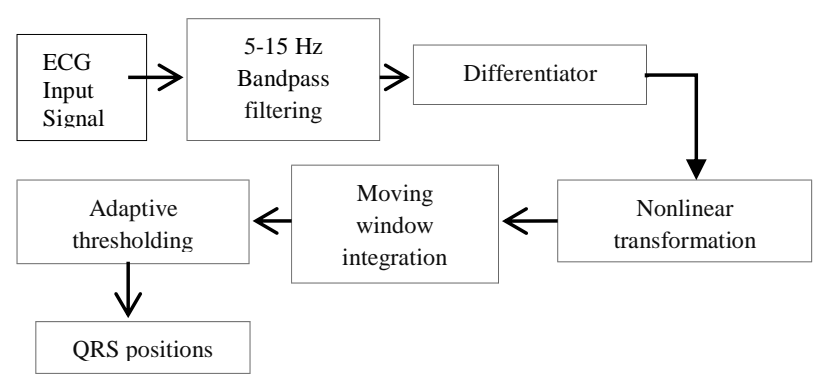

Figure 1.The five operations of the Pan-Tompkins algorithm.

In [2], a QRS detection based on fractional-order operators is developed where the same filtering steps like Pan-Tompkins were used. Xue et al. [27] proposed the use of an Artificial Neural Network (ANN) noise suppression filter and a matching filter adapted with an ANN classifier to detect QRS waves. Ruha et al. [19] employed an amplitude gain with a cascade of filters and a noise suppression filter with a matching filter for QRS positions detection. Ravanshad et al. [18] gave special attention to the energy consumption, where they exploited the efficiency of an analog to digital converter for noise suppression; then they introduced a decision stage to extract QRS positions by level-crossing. Finally, they employed decision rules and two adaptive thresholds of an adapted period to reject fast high T-waves confused with the QRS complex. Karimipour and Homaeinezhad [11] proposed a new QRS detection algorithm; they used wavelets for denoising and detected QRS using curves interpretation. Recently, Castells-Rufas and Carrabina [3] proposed MaMeMi 
filter, justified by low computational complexity, the authors make use of multiple filters and decision rules with an adaptive threshold for QRS detection. Saadi et al. [20] designed an optimized time-domain algorithm evaluated on a large dataset, and then they implemented the developed algorithm on an embedded e-Patch system. Recently, Yakut and Bolat [28] proposed an improved QRS detector with a low computational load. Tekeste et al. [24] developed a low power consumption system for QRS detection and ECG compression. Mourad and Fethi [12] detect QRS complex from wavelet coefficients after filtering for denoising. Nguyen et al. [15] proposed the triangle template matching for the determination of the QRS positions. Xiang et al. [26] used two Convolutional Neural Networks and an MLP for the estimation of the QRS positions.

All of the existing algorithms in the literature that detect the QRS complex in the time domain select the filtering parameters empirically. As an exception, Poli et al. [17] proposed three different architectures for QRS detection. They used linear and nonlinear filters to enhance the QRS complex and a decision stage. The filters coefficients and the parameters of the decision stage were optimized by genetic algorithms. However, the performance achieved by the optimization approach of [17] was poor as it delivered too much false detection using the benchmark MIT-BIH dataset.

We think that the performance of these algorithms could be enhanced if the filtering parameters were optimized. In particular, many parameters of the PanTompkins algorithm [16] are selected empirically. For example, the cut off frequencies of the band pass filter were determined by knowledge of an estimate band frequency of different sources such as muscle artifacts and stress. Also, the length of the averaging window and the parameters of the threshold are selected empirically. Since the problem at hand cannot be formulated in a closed-form formula, traditional optimization methods that rely on the computation of the gradient cannot be used. Therefore, we resort to evolutionary computational optimization methods. Among these methods, Particle Swarm Optimization (PSO) has gained a lot of popularity during the last two decades. The popularity of the PSO may be explained by its simplicity, few code lines, and it provides potential solutions in many complex situations. Thus, we propose to use the particle swarm optimization algorithm to look for the best values of the parameters of the popular Pan-Tompkins algorithm such as cut off frequencies of the band pass filter, the length of the averaging window, the threshold, etc., to improve its capability of detection for the QRS complexes.

\section{The Pan-Tompkins Algorithm}

Since our contribution is about improving the PanTompkins algorithm, we start by describing the main steps involved in this algorithm. The main steps of the original Pan-Tompkins algorithm are depicted in Figure 1. The algorithm comprises five steps which we will briefly describe in the following. The bandpass filter eliminates noise from the input raw ECG signal. The differentiator is introduced to detect abrupt variations in the signal. The nonlinear transformation is the square of the differentiated signal used to reduce the amplitudes of the $\mathrm{T}$ and $\mathrm{P}$ waves compared to $\mathrm{R}$ peak amplitude wave (after normalization) and to make the values of the signal positive. The aim of the moving window integration is to make the $\mathrm{P}, \mathrm{Q}, \mathrm{R}, \mathrm{S}$, and $\mathrm{T}$ peaks appear together in a unique peak in order to reduce the number of false detections.

The decision strategy relies on the use of two main parameters which are the signal level and the noise level. The former is computed from a running estimation of the signal level whereas the latter is computed from a running estimation of the noise level. In particular, the first type of thresholding which is a running level of the separation between the amplitudes of the signal and the amplitudes of noise is formed by combining the estimated levels of the signal and noise. This type is used in normal cases. When no $\mathrm{R}$ peak is detected in a long period of time using the first type of thresholding, a search back procedure is launched with another type of thresholding. This second type of thresholding uses new rules for $\mathrm{R}$ peak detection.

In the training phase of our optimization algorithm, we estimate the threshold levels of the signal and noise from the few first seconds of the ECG input signal. First, we search for all the peaks present in this time interval and the minimum interval between them. We decide that a peak is an $\mathrm{R}$ wave if its amplitude is greater than the threshold level, and we update the level of the signal. Otherwise, if the amplitude of the peak is greater than the threshold level and the mean of amplitudes in this region is less than or equal to a certain percentage of the mean of amplitudes in the region of the previously detected $\mathrm{R}$ wave, and the interval between the current peak and the last detected $\mathrm{R}$ peak is within a certain value, then the current peak is a $\mathrm{T}$ wave and we update the noise level. If the amplitude of the current peak is less than the level of the search back threshold, it is a noise peak; we update the level of noise then. At each peak, we test if a search back is needed, and we update the threshold levels according to the levels of the signal and noise.

The search back procedure checks if the current RR interval is within a predefined interval. Otherwise, we reduce the level of the signal, if the current RR interval is greater than a predefined limit, then a search back is needed, we update the level of the signal. If the maximum amplitude between the last detected peak and the current peak is greater than or equal to the threshold level of the search back procedure, the corresponding position is considered as an $\mathrm{R}$ peak. 


\section{The Particle Swarm Optimization (PSO)}

PSO is a heuristic algorithm, inspired by fish flocking and birds flying. It has been used in a variety of optimization problems and usually delivers interesting results. It involves many characteristics which are common to heuristics search algorithms such as initial population, calculation of the current outputs, and mutation of the population to potentially reach better solutions.

The PSO algorithm has many advantages; among them, we cite fast convergence, simplicity of implementation using only a few lines of code, and a reduced number of parameters. The potential solutions of the population of the PSO are called particles; they are usually initialized with random values. Each particle $\left(p_{i}\right)$ has its proper knowledge of its environment, represented by the present values which are the input values to the fitness function, and the values which are the best set of parameters that give the best output value $\left(p_{b i}\right)$ for a certain particle. The present values of a particle are adjusted at each generation by the velocity vector $\left(\mathrm{v}_{i}\right)$.

The algorithm possesses a global best $\left(\mathrm{p}_{g}\right)$ which is updated during each generation; it represents the best solution found so far, which is a social knowledge. The steps involved in the PSO algorithm are illustrated in the block diagram of Figure 2.

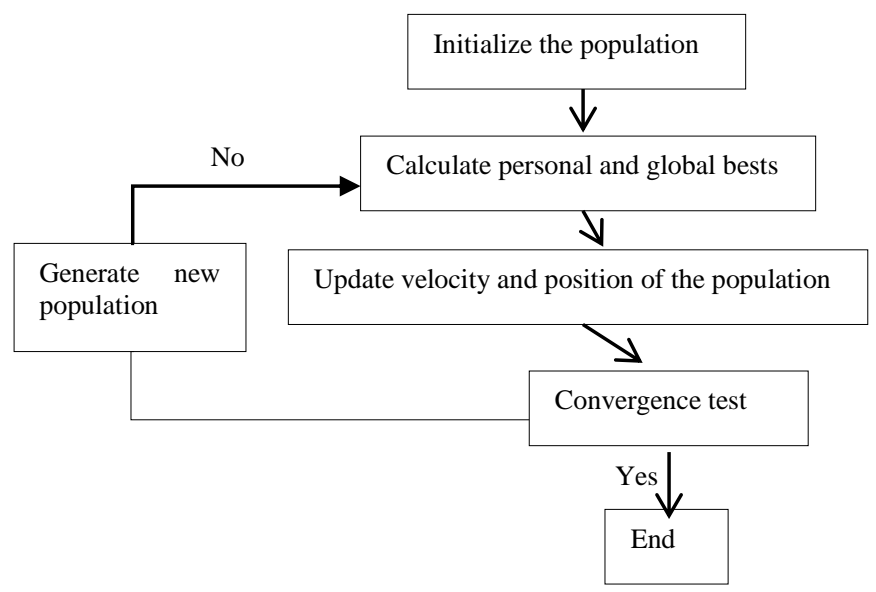

Figure 2. The steps involved in the PSO algorithm.

During each generation, the PSO algorithm updates its velocity vector and particles positions according to the following two equations.

$$
\begin{gathered}
\mathbf{v}_{i}(t+1)=w \mathbf{v}_{i}(t)+c_{1} \cdot \mathbf{r}_{1}(t)\left(\mathrm{p}_{b i}(t)-\mathrm{p}_{i}(t)\right)+ \\
c_{2} \cdot \mathbf{r}_{2}(t)\left(\mathrm{p}_{g}(t)-\mathrm{p}_{i}(t)\right) \\
\mathrm{p}_{i}(t+1)=\mathrm{p}_{i}(t)+\mathrm{v}_{i}(t)
\end{gathered}
$$

$w$ stands for the inertia factor introduced as a regularization factor in (1) to prevent premature convergence. $r_{1}$ and $r_{2}$ are random numbers drawn from a uniform distribution in [0,1], $c_{1}$ and $c_{2}$ are constants.

\section{The Proposed Optimization Approach}

\subsection{PSO Setup}

As mentioned above the parameters of the PanTompkins are selected empirically. In particular, the coefficients involved in the design of the low pass and high pass filters are chosen to be integers in order to reduce the computation load of the algorithm and make it appropriate for real-time implementation on microprocessors.

Also, the frequency response of the final resulting band pass filter is approximately $5-11 \mathrm{~Hz}$ [16]. We think that this range is so narrow to represent the QRS wave complex which needs a wider range to represent its abrupt change in the time domain. Hence, we propose a new procedure to look for optimal values of the Pan-Tompkins algorithm which allows better QRS detection. For this purpose, we formulate the optimization problem as a space of parameters searched by means of the PSO algorithm.

Given the raw ECG signal which contains 24 hours of recording of the MIT-BIH Dataset, we applied the Pan-Tompkins algorithm with initial values of [16]. Our PSO-based algorithm tries to change the values of the Pan-Tompkins parameters to look for potential new best values for the next iteration. The main steps of the proposed optimization procedure are depicted in Figure 3. The proposed scheme is driven by the performance of the ability of the Pan-Tompkins algorithm to detect the QRS complex. The PSO adjusts the parameters of the Pan-Tompkins algorithm in such a way that the detection of the QRS complex improves with the course of time.

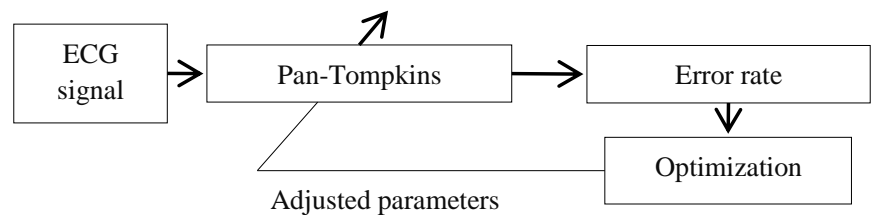

Figure 3. Flowchart showing the steps involved in the proposed optimization scheme.

In general, the most challenging thing in the application of the PSO is finding the appropriate fitness function. In our optimization scheme which relies on the PSO algorithm, in order to explore the solution space which depends on many parameters, we investigated three different fitness functions.

The first fitness function is the total error, the second and the third ones use the sensitivity and the specificity with different weights. In particular, the first fitness function is given by

$$
F_{1}(X)=\frac{F P+F N}{T P+F P+F N}
$$

Where $X$ is the vector of the parameters, here we consider only the total detection error. In Equation (3), TP stands for true positive beats, which is the number of beats effectively present in the record and effectively detected by the algorithm. False Positive 
(FP) is the number of beats detected by the algorithm, but not effectively present in the record. False Negative (FN) is the number of beats not detected by the algorithm, but present in the record. The second fitness function is given by

$$
\mathrm{F}_{2}(\mathrm{X})=(100-\mathrm{Se})^{2}+(100-\mathrm{Sp})^{2}
$$

Which is the Euclidian distance of the error based on the Sensitivity (Se) and the Specificity ( $\mathrm{Sp}$ ).

The third fitness function is given by

$$
\begin{gathered}
\mathrm{F}_{3}(\mathrm{X})=0.75 *(100-\mathrm{Se})^{2}+ \\
0.25 *(100-\mathrm{Sp})^{2}
\end{gathered}
$$

Which is the weighted Euclidian distance of the error. In Equation (5) more importance is given to the sensitivity as compared to the specificity due to the major attention given to this ratio by medical practitioners. In Equations (4) and (5); the sensitivity and the specificity are defined as

$$
\begin{gathered}
S e=\frac{T P}{T P+F N} \\
S p=\frac{T P}{T P+F P}
\end{gathered}
$$

Sensitivity expressed by Equation (6) measures the ability of our detector to extract QRS complex positions. Indeed, the sensitivity increases with the reduction of the number of missed beats. Likewise, the specificity described by Equation (7) measures the ability of our detector to reduce false detections; this is reflected by an increase in specificity when FP decreases. Equations (6) and (7) are exploited in Equations (4) and (5) with different weights to measure their effectiveness in driving the optimization algorithm.

Applying our proposed procedure using the adopted three fitness functions on the MIT-BIH dataset has led to the results shown in Figure 4. It is the variation of the fitness value as a function of the iteration number. We can see that $F_{3}(X)$ converges faster than $F_{1}(X)$ and $F_{2}(X)$. Moreover, from Figure 5, Equations (4) and (5) minimize better the error rate, and Equation (5) converges much faster than Equation (4). Furthermore, from Figures 6 and 7, which represent the evolution of the sensitivity and specificity, respectively, it is clear that using Equation (5) for optimization allows both the sensitivity and the specificity increasing faster, while Equation (3) does not achieve a considerable improvement of the specificity which gives rise to higher error rate than the other variants.

In summary, after extensive simulations, for the rapid convergence, and for the best approximation of the minimum error rate, we selected (5) as a fitness function for our proposed optimization approach.

\subsection{Procedure}

The main steps of the proposed scheme are detailed in the following algorithm.

\section{- Initialization}

1. Set initial values for different parameters of the Pan-Tompkins algorithm (e.g., low cut off frequency, high cut off frequency, filter order, number of samples for the integration window, etc.,) to be the initial population of the PSO algorithm.

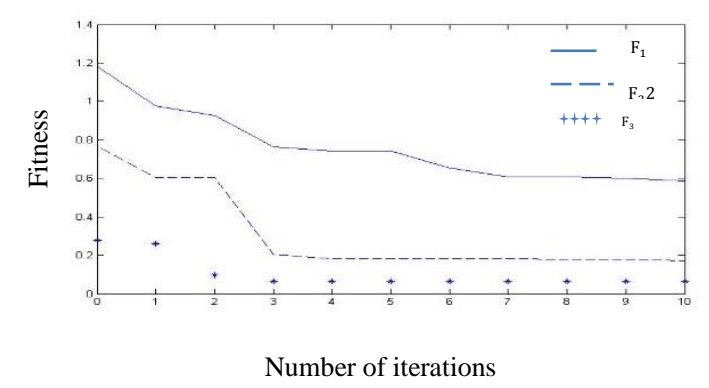

Figure 4. The fitness function vs. the number of iterations.

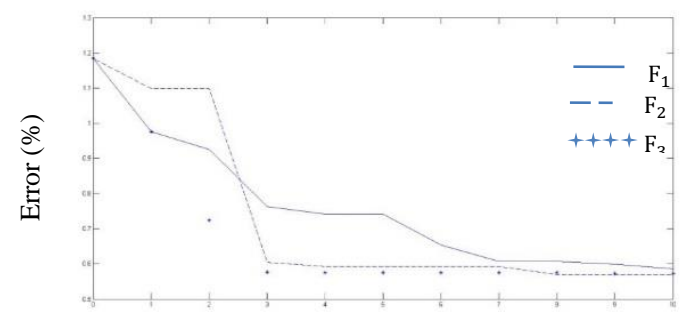

Number of iterations

Figure 5. The total error vs. the number of iterations.

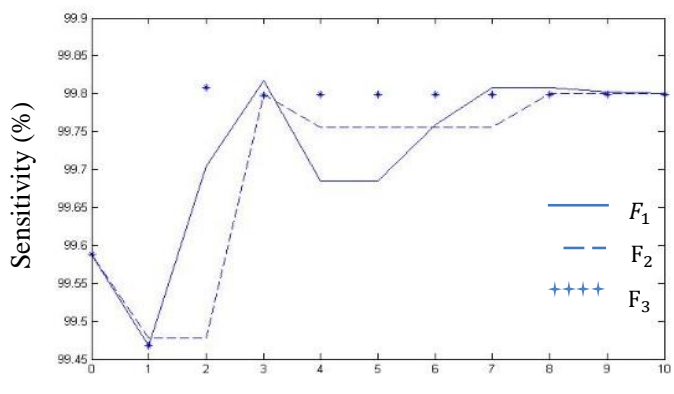

Number of iterations

Figure 6 . The sensitivity vs. the number of iterations.

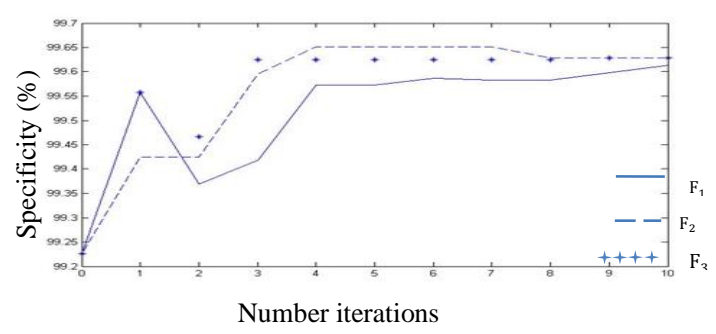

Figure 7. The specificity vs. the number of iterations.

2. Choose a random initial value for the velocity vector. For each particle evaluate the fitness function given by (5)

3. Store the position of each particle and label it as the best local position.

4. Save the position of the particle with the largest fitness function value as the best global position.

- Search process (parameters updating) 
1. Update the values of the velocity of each particle using (1).

2. Update the position of each particle using (2).

3. Using the new particle coordinates compute the new fitness function of each particle.

4. Update the best local positions and save the coordinates of the particle with the minimum fitness function as the best global position.

- Convergence check

1. Repeat the "search process" steps until the user pre-defined number of iterations or prede fined accuracy is reached (convergence check).

\section{Experimental Results and Discussions}

\subsection{Dataset Description}

For the sake of the assessment of the proposed optimization procedure, we conducted experiments on the basis of the benchmark MIT-BIH dataset available online at [21]. It consists of 48 records acquired from 47 subjects. Each record is 30 minutes long. The total number of beats used in our experiments is 109494 .

It covers a wide range of arrhythmia; hence, it contains a wide range of QRS shapes which represent a big challenge for any detection system. The dataset was annotated independently by two experts.

\subsection{Experimental Setup}

The PSO empirical parameters were selected according to the following configuration: The size of the swarm was fixed to 20 and the number of iteration was set to 550. The number of iterations has been set after many trials where it has been observed that the proposed algorithm converges after 500 iterations. The inertia weight was set to $w=0.4$ and both the cognitive coefficient $\mathrm{c} 1$ and the social coefficient $\mathrm{c} 2$ are set to 1 .

It is worth noting that the band-pass filter used in our optimization scheme is a Finite Impulse Response (FIR) filter. We preferred the FIR filter because of its intrinsic characteristics of stability. Though Infinite Impulse Response (IIR) filters design results in a lower filter order, it is necessary to check for stability every time the filter coefficients are updated.

It is common to adopt as measures for the performance assessment of any QRS detection algorithm three measures, which are the total error represented by Equation (3), the sensitivity represented by Equation (6), and the specificity given by Equation (7).

\subsection{Results}

The obtained results at convergence for the optimized parameters of the Pan-Tompkins algorithm are summarized in Table 1. We note that most of the original Pan-Tompkins parameters were not optimal as the obtained results confirm. This is because the parameters were selected empirically. Our claim is supported by the results obtained by the proposed optimization scheme evaluated on the MIT-BIH dataset shown in Table 2. As expected, the obtained band-pass filter cut off frequencies by our procedure $[4,24] \mathrm{Hz}$ are wider than the ones of the original PanTompkins algorithm, [5, 15] Hz. This allowed detecting many QRS waves that were missed by the Pan-Tompkins algorithm. Overall, our scheme failed in detecting 185 beats only. Whereas, the optimization scheme converged to the same length of the averaging window (30 samples) and the same number of the average RR intervals (8 intervals).

Table 1. Obtained Parameters of the Pan-Tompkins Algorithm at Convergence Using the Proposed Optimization Scheme. Initial Value Refers to The Value of The Parameter In Pan-Tompkins.

\begin{tabular}{|c|c|}
\hline Record & Nb. of beats \\
\hline The low cutoff frequency of the bandpass filter & $4(5)(\mathrm{Hz})$ \\
\hline The high cutoff frequency of the bandpass filter & $24(15)(\mathrm{Hz})$ \\
\hline Order of the FIR bandpass filter & 250 (3 IIR) \\
\hline Window length of the integrator & 30 (30) (samples) \\
\hline Minimum two successive peaks distance & 99 (72) (samples) \\
\hline Length of the window RR averaging & 8 (8) (samples) \\
\hline $\begin{array}{c}\text { Search back signal level percentage from normal signal } \\
\text { level }\end{array}$ & $1.5326(2)$ \\
\hline \begin{tabular}{|c|} 
Current peak update factor in the search back \\
procedure
\end{tabular} & $0.4427(0.25)$ \\
\hline Signal level update factor in the search back procedure & $0.7546(0.75)$ \\
\hline $\begin{array}{l}\text { Distance between the current sample and the last } \\
\text { detected } \mathrm{R} \text { peak in the search back procedure }\end{array}$ & 139 (72) (samples) \\
\hline $\begin{array}{l}\text { Distance between the current sample and the current } \\
\text { peak in the search back procedure }\end{array}$ & 83 (72) (samples) \\
\hline RR LOW LIMIT percentage from RR AVERAGE2 & $90.7 \%(92 \%)$ \\
\hline RR HIGH LIMIT percentage from RR AVERAGE2 & $116.74 \%(116 \%)$ \\
\hline RR MISSED LIMIT percentage from RR AVERAGE2 & $160.44 \%(166 \%)$ \\
\hline \begin{tabular}{|c|} 
Distance between the current peak and the last detected \\
$\mathrm{R}$ peak
\end{tabular} & 119 (130) (samples) \\
\hline \begin{tabular}{|l|} 
Length of the current peak region \\
\end{tabular} & 31 (27) (samples) \\
\hline Factor of mean amplitudes of the $\mathrm{R}$ peak region & $0.5634(0.5)$ \\
\hline Current peak update factor for noise level estimation & $0.2316(0.125)$ \\
\hline Signal level update factor for noise level estimation & $1.8228(0.875)$ \\
\hline Current peak update factor for signal level estimation & $0.1074(0.125)$ \\
\hline Signal level update factor for signal level estimation & $0.8673(0.875)$ \\
\hline $\begin{array}{c}\text { Second current peak update factor for noise level } \\
\text { estimation }\end{array}$ & $0.1514(0.125)$ \\
\hline $\begin{array}{c}\text { Second signal level update factor for noise level } \\
\text { estimation }\end{array}$ & $0.8745(0.875)$ \\
\hline The low cutoff frequency of the bandpass filter & $4(5)(\mathrm{Hz})$ \\
\hline The high cutoff frequency of the bandpass filter & $24(15)(\mathrm{Hz})$ \\
\hline Order of the FIR bandpass filter & 250 (3 IIR) \\
\hline Window length of the integrator & 30 (30) (samples) \\
\hline Minimum two successive peaks distance & 99 (72) (samples) \\
\hline Length of the window RR averaging & 8 (8) (samples) \\
\hline $\begin{array}{c}\text { Search back signal level percentage from normal signal } \\
\text { level }\end{array}$ & $1.5326(2)$ \\
\hline \begin{tabular}{|l|} 
Current peak update factor in the search back \\
procedure
\end{tabular} & $0.4427(0.25)$ \\
\hline Signal level update factor in the search back procedure & $0.7546(0.75)$ \\
\hline $\begin{array}{l}\text { Distance between the current sample and the last } \\
\text { detected R peak in the search back procedure }\end{array}$ & 139 (72) (samples) \\
\hline $\begin{array}{l}\text { Distance between the current sample and the current } \\
\text { peak in the search back procedure }\end{array}$ & 83 (72) (samples) \\
\hline RR LOW LIMIT percentage from RR AVERAGE2 & $90.7 \%(92 \%)$ \\
\hline RR HIGH LIMIT percentage from RR AVERAGE2 & $116.74 \%(116 \%)$ \\
\hline RR MISSED LIMIT percentage from RR AVERAGE2 & $160.44 \%(166 \%)$ \\
\hline $\begin{array}{l}\text { Distance between the current peak and the last detected } \\
\text { R peak }\end{array}$ & 119 (130) (samples) \\
\hline
\end{tabular}


Table 2. QRS Detection Results by the Proposed Optimization Scheme. Here Error $(\%)=100 *(\mathrm{FP}+\mathrm{FN}) /(\mathrm{TP}+\mathrm{FP}+\mathrm{FN})$.

\begin{tabular}{|c|c|c|c|c|c|c|c|}
\hline Record & Nb. of beats & TP & FP & FN & Error (\%) & Sp \% & Se \% \\
\hline $100 \mathrm{~m}$ & 2273 & 2273 & 0 & 1 & 0.04 & 100 & 99.96 \\
\hline $101 \mathrm{~m}$ & 1865 & 1865 & 0 & 0 & 0 & 100 & 100 \\
\hline $102 \mathrm{~m}$ & 2187 & 2187 & 0 & 0 & 0.0 & 100 & 100 \\
\hline $103 \mathrm{~m}$ & 2084 & 2084 & 0 & 0 & 0.0 & 100 & 100 \\
\hline $104 \mathrm{~m}$ & 2229 & 2224 & 5 & 0 & 0.22 & 99.78 & 100 \\
\hline $105 \mathrm{~m}$ & 2572 & 2551 & 21 & 1 & 0.86 & 99.18 & 99.96 \\
\hline $106 \mathrm{~m}$ & 2027 & 2027 & 0 & 0 & 0.0 & 100 & 100 \\
\hline $107 \mathrm{~m}$ & 2137 & 2137 & 0 & 3 & 0.14 & 100 & 99.86 \\
\hline $108 \mathrm{~m}$ & 1763 & 1755 & 8 & 0 & 0.45 & 99.54 & 100 \\
\hline $109 \mathrm{~m}$ & 2532 & 2532 & 0 & 0 & 0 & 100 & 100 \\
\hline $111 \mathrm{~m}$ & 2124 & 2124 & 0 & 1 & 0.05 & 100 & 99.95 \\
\hline $112 \mathrm{~m}$ & 2539 & 2539 & 0 & 0 & 0.0 & 100 & 100 \\
\hline $113 \mathrm{~m}$ & 1795 & 1791 & 4 & 1 & 0.28 & 99.78 & 99.94 \\
\hline $114 \mathrm{~m}$ & 1879 & 1875 & 4 & 1 & 0.27 & 99.79 & 99.95 \\
\hline $115 \mathrm{~m}$ & 1953 & 1953 & 0 & 1 & 0.05 & 100 & 99.95 \\
\hline $116 \mathrm{~m}$ & 2412 & 2407 & 0 & 14 & 0.58 & 100 & 99.42 \\
\hline $117 \mathrm{~m}$ & 1535 & 1535 & 0 & 0 & 0 & 100 & 100 \\
\hline $118 \mathrm{~m}$ & 2278 & 2277 & 1 & 0 & 0.04 & 99.96 & 100 \\
\hline $119 \mathrm{~m}$ & 1987 & 1986 & 0 & 0 & 0 & 100 & 100 \\
\hline $121 \mathrm{~m}$ & 1863 & 1863 & 0 & 1 & 0.05 & 100 & 99.95 \\
\hline $122 \mathrm{~m}$ & 2476 & 2476 & 0 & 0 & 0 & 100 & 100 \\
\hline $123 \mathrm{~m}$ & 1518 & 1518 & 0 & 0 & 0.0 & 100 & 100 \\
\hline $124 \mathrm{~m}$ & 1619 & 1619 & 0 & 0 & 0.0 & 100 & 100 \\
\hline $200 \mathrm{~m}$ & 2601 & 2599 & 2 & 2 & 0.15 & 99.92 & 99.92 \\
\hline $201 \mathrm{~m}$ & 1963 & 1960 & 3 & 10 & 0.66 & 99.85 & 99.49 \\
\hline $202 \mathrm{~m}$ & 2136 & 2136 & 0 & 3 & 0.14 & 100 & 99.86 \\
\hline $203 \mathrm{~m}$ & 2980 & 2977 & 3 & 17 & 0.67 & 99.9 & 99.43 \\
\hline $205 \mathrm{~m}$ & 2656 & 2656 & 0 & 3 & 0.11 & 100 & 99.89 \\
\hline $207 \mathrm{~m}$ & 1860 & 1856 & 4 & 5 & 0.48 & 99.79 & 99.73 \\
\hline $208 \mathrm{~m}$ & 2955 & 2951 & 2 & 10 & 0.41 & 99.93 & 99.66 \\
\hline $209 \mathrm{~m}$ & 3005 & 3005 & 0 & 0 & 0 & 100 & 100 \\
\hline $210 \mathrm{~m}$ & 2650 & 2649 & 1 & 7 & 0.3 & 99.96 & 99.74 \\
\hline $212 \mathrm{~m}$ & 2748 & 2748 & 0 & 0 & 0 & 100 & 100 \\
\hline $213 \mathrm{~m}$ & 3251 & 3251 & 0 & 1 & 0.31 & 100 & 99.97 \\
\hline $214 \mathrm{~m}$ & 2262 & 2262 & 0 & 1 & 0.04 & 100 & 99.96 \\
\hline $215 \mathrm{~m}$ & 3363 & 3365 & 0 & 3 & 0.09 & 100 & 99.91 \\
\hline $217 \mathrm{~m}$ & 2208 & 2206 & 0 & 0 & 0 & 100 & 100 \\
\hline $219 \mathrm{~m}$ & 2148 & 2158 & 0 & 0 & 0 & 100 & 100 \\
\hline $220 \mathrm{~m}$ & 2048 & 2048 & 0 & 0 & 0 & 100 & 100 \\
\hline $221 \mathrm{~m}$ & 2427 & 2427 & 0 & 1 & 0 & 100 & 100 \\
\hline $222 \mathrm{~m}$ & 2483 & 2463 & 19 & 0 & 0.77 & 0.24 & 100 \\
\hline $223 \mathrm{~m}$ & 2605 & 2605 & 0 & 0 & 0.0 & 100 & 100 \\
\hline $228 \mathrm{~m}$ & 2053 & 2047 & 8 & 1 & 0.44 & 99.61 & 99.95 \\
\hline $230 \mathrm{~m}$ & 2256 & 2256 & 0 & 0 & 0.0 & 100 & 100 \\
\hline $231 \mathrm{~m}$ & 1571 & 1998 & 0 & 0 & 0.0 & 100 & 100 \\
\hline $232 \mathrm{~m}$ & 1780 & 1768 & 11 & 0 & 0.73 & 99.38 & 100 \\
\hline $233 \mathrm{~m}$ & 3079 & 3079 & 0 & 1 & 0.03 & 100 & 99.97 \\
\hline $234 \mathrm{~m}$ & 2753 & 2752 & 1 & 0 & 0.04 & 99.96 & 100 \\
\hline Total & 109494 & 109397 & 97 & 88 & 0.169 & 99.91 & 99.92 \\
\hline
\end{tabular}

For a fair comparison with the existing methods, we compared our optimization scheme with the state-ofthe-art methods. As shown in Table 3, our optimization scheme outperforms all time-domain methods including the well-known Pan-Tompkins algorithm when using approximately the same number of test beats. The only exception which produced the best result $(0.15 \%$ of error) used only 101579 beats, which is less than our total umber of test beats by more than 7900 beats. It is worth noting that our optimization produced $99.92 \%$ of sensitivity and $99.91 \%$ of predictivity.

We developed this optimization approach to enhance the monitoring of heart disease which is the leading cause for death and to contribute to the improvement of the detection process. Generally, repeated failures of the QRS detector influence the disease detection process and this has a bad influence on the diagnosis result. The obtained results for QRS detection presented in this manuscript give us more confidence in the computerized methods for disease identification.
Table 3. Comparison of the Performance of the Proposed Scheme with State-of-the-Art Methods. Here Error $(\%)=100 *(\mathrm{FP}+\mathrm{FN}) /(\mathrm{TP}+\mathrm{FP}+\mathrm{FN}$.

\begin{tabular}{|c|c|c|c|c|}
\hline Method & Nb of beats & Error & Se $\%$ & Sp \% \\
\hline Geometrical matching [23] & 60431 & 2.92 & 97.94 & 99.13 \\
\hline Zero crossing $[18]$ & 109428 & 1.71 & 97.44 & 99.13 \\
\hline $\begin{array}{l}\text { Short Time Fourier Transform } \\
{[25]}\end{array}$ & 109982 & 1.3 & 99.1 & 99.6 \\
\hline Moving average [4] & 102654 & 0.96 & 99.6 & 99.78 \\
\hline $\begin{array}{l}\text { STFT using Adap. Threshold } \\
\text { [22] }\end{array}$ & 109011 & 0.93 & 99.56 & 99.52 \\
\hline MaMeMi filter [3] & 109494 & 0.88 & 99.68 & 99.44 \\
\hline AdaptativeThresholding [6] & 109949 & 0.72 & 99.54 & 99.74 \\
\hline Pan-Tompkins [16] & 109809 & 0.68 & 99.76 & 99.56 \\
\hline Hamilton [7] & 109267 & 0.54 & 99.69 & 99.77 \\
\hline Low cost $[15]$ & 109494 & 0.49 & 99.8 & 99.71 \\
\hline $\begin{array}{c}\text { Morphological filtering(VLSI) } \\
{[30]}\end{array}$ & 109510 & 0.43 & 99.76 & 99.82 \\
\hline $\begin{array}{c}\text { Multiscale morphological } \\
\text { filtering [29] }\end{array}$ & 109510 & 0.39 & 99.81 & 99.8 \\
\hline Two moving averages [5] & 109985 & 0.35 & 99.78 & 99.87 \\
\hline CNN Detector [26] & 105078 & 0.32 & 99.77 & 99.91 \\
\hline Efficient Detection [12] & 106310 & 0.29 & 99.76 & 99.95 \\
\hline Fractional order operator [2] & 107632 & 0.29 & 99.86 & 99.86 \\
\hline $\begin{array}{c}\text { Shannon energy envelope } \\
\text { estimation [14] }\end{array}$ & 109809 & 0.2 & 99.93 & 99.88 \\
\hline $\begin{array}{c}\text { Proposed optimized } \\
\text { Pan-Tompkins algorithm }\end{array}$ & 109494 & 0.17 & 99.92 & 99.91 \\
\hline Wavelet Detection [13] & 101579 & 0.15 & 99.89 & 99.94 \\
\hline
\end{tabular}

Concerning the execution time, the proposed scheme requires 1.3 seconds to detect 2273 beats from the $100 \mathrm{~m} \mathrm{MIT/BIH} \mathrm{record,} \mathrm{which} \mathrm{has} \mathrm{a} \mathrm{duration} \mathrm{of} 30$ minutes and contains 650000 samples, using an Intel i5 M480 $2.67 \mathrm{GHz}$ with 4GB of RAM running on MATLAB software, corresponding to $57.19 \mathrm{~ms}$ to detect a beat, or $2 \mu$ s to process a sample, making our scheme suitable for real-time and embedded systems. Our detector outperforms all existing methods except two works, Nguyen et al. [15] with 0.16 seconds to process 30 minutes of recording, and 0.43 seconds at mean reported by Elgendi [5]. However, the proposed scheme is superior in detection and accuracy. On the benchmark MIT arrhythmia, our algorithm generates $0.17 \%$ of DER error, where Nguyen et al. [15] reported $0.49 \%$ of error. Also, in terms of sensitivity and specificity, the work of Elgendi [5] is inferior to ours with $99.78 \%$ of sensitivity and $99.87 \%$ of specificity, where our detector achieved $99.92 \%$ of sensitivity and $99.91 \%$ of specificity.

For the other knowledge works, no method is faster than our scheme. For instance, the method of Bal et al. [1] spends 1.92 seconds, Hashim et al. [9] spends 2.97 seconds, Xiang et al. [26] spends 14.53 seconds, and Karimipour et al. [11] spends 18.18 seconds to process 30 minutes ECG record, respectively. Also, the algorithm of Kholkhal et al. [12] requires 1476 seconds to detect 15027 beats, which means that it needs 223.26 seconds to process 2273 beats. Finally, the work of Saadi et al. [20] requires 2.3 hours to process 4271185 samples, which is equivalent to 1260.07 seconds to process 650000 samples. Therefore, our scheme is the best and is suitable for low cost and mobile devices. 


\section{Conclusions}

In this paper, we proposed an optimization scheme that exploits the features of the PSO algorithm to search for the optimized parameters of the well-known PanTompkins algorithm. Though the PSO algorithm is very simple, it allowed us to find a set of optimal parameters for enhancing the QRS complex and then setting the value of the thresholding to minimize then umber of false detection all in only one global optimization approach. In particular, the proposed scheme efficiently determines a set of parameters using an optimization algorithm driven by sensitivity and specificity. The main contribution of this paper is the generation of the parameters of the detection algorithm automatically. Whereas, the parameters of other algorithms are set empirically.

To the best of our knowledge, the PSO algorithm has not been used to optimize the parameters of the QRS detection scheme. Our proposed scheme outperforms the state-of-the-art time-domain algorithms that are published in the literature so far.

The high detection rate achieved by our proposed approach may help clinicians in improving the heartbeat segmentation process which is timeconsuming if done manually. Further work could be something related to developing a parallel scheme to reduce the processing time.

\section{Acknowledgment}

We thank the Algerian direction of research (DGRSDT) for providing the financial support, under Project contract numberA01L08UN35020150002

\section{References}

[1] Bal R. and Kumar A., "Improved QRS Detector Using Parallel based Hybrid Mamemi Filter," International Journal of Image, Graphics and Signal Processing, vol. 9, no. 3, pp. 55-61, 2017.

[2] Benmalek M. and Charef A., "Digital FractionalOrder Operators for R-Wave Detection in TheElectrocardiogram," IET Signal Process, vol. 3, no. 5, pp. 381-391, 2008.

[3] Castells-Rufas D. and Carrabina J., "Simple RealTime QRS Detector with the Mamemi Filter," Biomedical Signal Processing and Control, vol. 21, pp. 137-145, 2015.

[4] Chen S., Chen H., and Chan H., "A Real-Time QRS Detection Method Based on MovingAveraging Incorporating with Wavelet Denoising," Computer Methods and Programs in Biomedicine, vol. 82, no. 3, pp. 187-195, 2006.

[5] Elgendi M., "Fast QRS Detection with an Optimized Knowledge-Based Method: Evaluation on 11 Standard ECG Databases," PlosONE, vol. 8, no. 9, 2013.

[6] Gutiérrez-Rivas R., García J., Marnane W., and
Hernández Á., "Novel Real-Time LowComplexity QRS Complex Detector Based on Adaptive Thresholding," IEEE Sensors Journal, vol. 15, no. 10, pp. 6036-6043, 2015.

[7] Hamilton P. and Tompkins W., "Quantitative Investigation of QRS Detection Rules Using the MIT/BIH Arrhythmia Database," IEEE Transactions on Biomedical Engineering, vol. BME-33, no. 12, pp. 1157-1165, 1986.

[8] Hammad M., Ibrahim M., and Hadhoud M., "A Novel Biometric Based on ECG Signals and Images for Human Authentification," The International Arab Journal of Information Technology, vol. 13, no. 6A, pp. 959-964, 2016.

[9] Hashim M., Hau Y., and Baktheri R., "Efficient QRS Complex Detection Algorithm Implementation on A Soc-Based Embedded System," Jurnal Teknologi, vol. 78, no. 7-5, pp. 49-58, 2016.

[10] Kadambe S., Murray R., and Bordeaux-Bartels G., "Wavelet transform-based QRS Complex Detector," IEEE Transactions on Biomedical Engineering, vol. 46, no. 7, pp. 838-848, 1999.

[11] Karimipour A. and Homaeinezhad M., "RealTime Electrocardiogram P-QRS-T DetectionDelineation Algorithm Based on QualitySupported Analysis of Characteristic Templates," Computers in Biology and Medicine, vol. 52, pp. 153-165, 2014.

[12] Mourad K. and Fethi B., "Efficient Automatic Detection of QRS Complexes in ECG Signal Based on Reverse Biorthogonal Wavelet Decomposition and Nonlinear Filtering," Measurement, vol. 94, pp. 663-670, 2016.

[13] Li C., Zheng C., and Tai C., "Detection of ECG Characteristic Points Using Wavelet Transforms," IEEE Transactions on biomedical Engineering, vol. 42, no. 1, pp. 21-28, 1995.

[14] Manikandan M. and Soman K., "A Novel Method for Detecting R-Peaks in Electrocardiogram (ECG) Signal," Biomedical Signal Processing and Control, vol. 7, no. 2, pp. 118-128, 2012.

[15] Nguyen T., Qin X., Dinh A., and Bui F., "Low Resource Complexity R-Peak Detection Based on Triangle Template Matching and Moving Average Filter," Sensors (Basel), vol. 19, no. 18, 2019.

[16] Pan J. and Tompkins W., "A Real-Time QRS Detector Algorithm," IEEE Transactions on Biomedical Engineering,vol. BME-32, no. 3, pp. 230-236, 1985.

[17] Poli R., Cagnoni S., and Valli G., "Genetic Design of Optimum Linear and Nonlinear QRS Detectors," IEEE Transactions on Biomedical Engineering, vol. 42, no. 11, pp. 1137-1141, 1995. 
[18] Ravanshad N., Rezaee-Dehsorkh H., Lotfi R., and Lian Y., "A Level Crossing Based QRS-Detection Algorithm for Wearable ECG Sensors," IEEE Transactions on Biomedical Engineering, vol. 18, no. 1, pp. 183-192, 2013.

[19] Ruha A., Sallinen S., and Nissila S., "A RealTime Microprocessor QRS Detector System with a 1-ms Timing Accuracy for the Measurement of Ambulatory HRV," IEEE Transactions on Biomedical Engineering, vol. 44, no. 3, pp. 159167, 1997.

[20] Saadi D., Tanev G., Flintrup M., Osmanagic A., Egstrup K., Hoppe K., Jennum J., Jeppesen J., Iversen H., and Sorensen H., "Automatic RealTime Embedded QRS Complex Detection for a Novel Patch-Type Electrocardiogram Recorder," IEEE Journal of Translational Engineering in Health and Medicine, vol. 3, pp. 1-12, 2015.

[21] Sedghamiz H., Complete Implementation of PanTompkins Software. Humanist, Hooman Sedghamiz, 2014.

[22] Shaik B., Naganjaneyulu G., Chandrasheker T., and Narasimhadhan A., "A Method for QRS Delineation Based on STFT using Adaptive Threshold," Procedia Computer Science, vol. 54, pp. 646-653, 2015.

[23] Suarez K., Silva J., Berthoumieu Y., Gomis P., and Najim M., "ECG Beat Detection Using A Geometrical Matching Approach," IEEE Transactions on Biomedical Engineering, vol. 54, no. 4, pp. 641-650, 2007.

[24] Tekeste T., Saleh H., Mohammad B., and Ismail M., "Ultra-Low Power QRS Detection and ECG Compression Architecture for IoT Healthcare Devices," IEEE Transactions on Circuits and Systems I: Regular Papers, vol. 66, no. 2, pp. 669 $-679,2018$.

[25] Uchaipichat N. and Sakonthawat I., "Development of QRS Detection using ShortTime Fourier Transform Based Technique," International Journal of Computer Applications, pp. 7-10, 2010.

[26] Xiang Y., Lin Z., and Meng J., "Automatic QRS Complex Detection Using Two-Level Convolutional Neural Network," Biomedical Engineering Online, vol. 17, no. 1, 2018.

[27] Xue Q., Hu Y., and Tompkins W., "NeuralNetwork-Based Adaptive Matched Filtering for QRS Detection," IEEE Transactions on Biomedical Engineering, vol. 39, no. 4, pp. 317329, 1992.

[28] Yakut Ö. and Bolat E., "An Improved QRS Complex Detection Method Having Low Computational Load," Biomedical Signal Processing and Control, vol. 42, pp. 230-241, 2018.

[29] Zhang F. and Lian Y., "QRS Detection Based on Multiscale Mathematical Morphology, for
Wearable ECG Devices in Body Area Networks," IEEE Transactions on Biomedical Circuits and Systems, vol. 3, no. 4, pp. 220-228, 2009.

[30] Zhang C. and BaeTae-Wuk., "VLSI Friendly ECG QRS Complex Detector for Body Sensor Networks," EEE Journal on Emerging and Selected Topics in Circuits and Systems, vol. 2, no. 1, pp. 52-59, 2012.

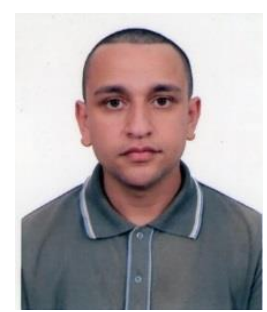

Mohamed Belkadi born on 1989 at Algiers, Algeria, he obtained his B.E in Computer Science in 2012 and M.S degree in 2014 from Medea University. Currently, he is a Ph.D. student at the University of Boumerdes, Algeria. His research interests include Digital Signal Processing, Biomedical Signal Processing, and Deep Learning.

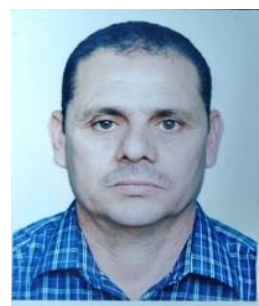

Abdelhamid Daamouche received the state engineer degree in Telecommunications from (INELEC), Boumerdes, Algeria, and the Magister degree from the University of Batna, and a Ph.D. degree in signal and image processing from the University of Boumerdes, in 2012. In 2003, he joined the University of Boumerdes as a Lecturer, and in 2019 became a Professor. His current research interests include pattern recognition, biomedical engineering, and remote sensing. 